\title{
Theoretical investigation of molecular properties of the first excited state of phenoxyl radical
}

\author{
Chi-Wen Cheng, Yuan-Pern Lee, and Henryk A. Witek \\ Institute of Molecular Science and Department of Applied Chemistry, National Chiao \\ Tung University, 30010 Hsinchu, Taiwan
}

\section{Auxiliary Materials:}

- Table S. Geometrical parameters in previous studies of the $\mathrm{X}^{2} \mathrm{~B}_{1}$ state of $\mathrm{C}_{6} \mathrm{H}_{5} \mathrm{O}$

- Table X1. Harmonic vibrational frequencies for the $\mathrm{X}^{2} \mathrm{~B}_{1}$ state of $\mathrm{C}_{6} \mathrm{H}_{5} \mathrm{O}$

- Table X2. Harmonic vibrational frequencies for the $\mathrm{X}^{2} \mathrm{~B}_{1}$ state of $\mathrm{C}_{6} \mathrm{D}_{5} \mathrm{O}$

- Table P1. B3LYP harmonic vibrational frequencies of $\mathrm{C}_{6} \mathrm{H}_{5} \mathrm{OH}$

- $\quad$ Table P2. B3LYP harmonic vibrational frequencies of $\mathrm{C}_{6} \mathrm{D}_{5} \mathrm{OD}$

- Table P3. CASSCF harmonic vibrational frequencies of $\mathrm{C}_{6} \mathrm{H}_{5} \mathrm{OH}$

- Table P4. CASSCF harmonic vibrational frequencies of $\mathrm{C}_{6} \mathrm{D}_{5} \mathrm{OD}$

- Table K. CASSCF excitation energies for the $\mathrm{A}^{2} \mathrm{~B}_{2}$ state of $\mathrm{C}_{6} \mathrm{H}_{5} \mathrm{O}$ 
Table S. Geometrical parameters from previous theoretical studies for the $\mathrm{X}^{2} \mathrm{~B}_{1}$ state of $\mathrm{C}_{6} \mathrm{H}_{5} \mathrm{O}$. Distances are given in $\AA$ and bonds, in degrees.

\begin{tabular}{|c|c|c|c|c|c|c|}
\hline & \multicolumn{6}{|c|}{$\mathrm{X}^{2} \mathrm{~B}_{1}$} \\
\hline & \multirow{2}{*}{$\frac{\text { CAS-MCSCF }}{\mathrm{DZV}}$} & \multirow{2}{*}{$\frac{\mathrm{UB}^{2} \mathrm{LYP}^{\mathrm{b}}}{\text { cc-pVTZ }}$} & \multicolumn{2}{|c|}{ CASSCF } & \multirow{2}{*}{$\frac{\text { UNOCAS }^{\mathrm{d}}}{6-31 \mathrm{G}^{*}}$} & \multirow{2}{*}{$\frac{\mathrm{UMP}^{\mathrm{e}}}{6-31 \mathrm{G}^{*}}$} \\
\hline & & & $6-311 G(2 d, p)^{c}$ & $6-31 \mathrm{G}^{* \mathrm{e}}$ & & \\
\hline$r_{\mathrm{C}_{1} \mathrm{O}}$ & 1.295 & 1.251 & 1.228 & 1.236 & 1.239 & 1.225 \\
\hline$r_{\mathrm{C}_{1} \mathrm{C}_{2}}$ & 1.441 & 1.449 & 1.454 & 1.455 & 1.452 & 1.461 \\
\hline$r_{\mathrm{C}_{2} \mathrm{C}_{3}}$ & 1.393 & 1.372 & 1.370 & 1.377 & 1.378 & 1.354 \\
\hline$r_{\mathrm{C}_{3} \mathrm{C}_{4}}$ & 1.418 & 1.405 & 1.411 & 1.414 & 1.413 & 1.404 \\
\hline$r_{\mathrm{C}_{2} \mathrm{H}_{1}}$ & 1.071 & 1.081 & 1.073 & 1.074 & 1.075 & 1.085 \\
\hline$r_{\mathrm{C}_{3} \mathrm{H}_{2}}$ & 1.071 & 1.082 & 1.074 & 1.075 & 1.075 & 1.086 \\
\hline$r_{\mathrm{C}_{4} \mathrm{H}_{3}}$ & 1.071 & 1.082 & 1.073 & 1.074 & 1.074 & 1.086 \\
\hline$\alpha_{\mathrm{C}_{6} \mathrm{C}_{1} \mathrm{C}_{2}}$ & 118.9 & 117.1 & 117.4 & 117.4 & 117.6 & 116.1 \\
\hline$\alpha_{\mathrm{C}_{1} \mathrm{C}_{2} \mathrm{C}_{3}}$ & 120.1 & 120.9 & 120.6 & 120.6 & 120.5 & 121.2 \\
\hline$\alpha_{\mathrm{C}_{2} \mathrm{C}_{3} \mathrm{C}_{4}}$ & 120.4 & 120.3 & 120.6 & 120.5 & 120.6 & 120.4 \\
\hline$\alpha_{\mathrm{C}_{1} \mathrm{C}_{2} \mathrm{H}_{1}}$ & $\ldots$ & 117.0 & 117.3 & 117.4 & 117.5 & 116.2 \\
\hline$\alpha_{\mathrm{C}_{4} \mathrm{C}_{3} \mathrm{H}_{2}}$ & $\ldots$ & 119.4 & 119.4 & 119.4 & 119.5 & 119.2 \\
\hline
\end{tabular}

${ }^{a}$ Ref. 11.

${ }^{\mathrm{b}}$ Ref. 6.

${ }^{\mathrm{c}}$ Ref. 5.

${ }^{\mathrm{d}}$ R. Liu and X. Zhou, J. Phys. Chem. 97, 9613 (1993).

${ }^{\mathrm{e}}$ Ref. 14. 
Table X1. Harmonic vibrational frequencies for the $\mathrm{X}^{2} \mathrm{~B}_{1}$ electronic state of $\mathrm{C}_{6} \mathrm{H}_{5} \mathrm{O}$ computed using the DFT/B3LYP and CASSCF methods. For description of the active space of CASSCF, see text. All values are given in $\mathrm{cm}^{-1}$.

\begin{tabular}{|c|c|c|c|c|c|c|c|c|}
\hline \multirow{2}{*}{ Symmetry } & \multirow{2}{*}{ Mode } & \multirow{2}{*}{ Exp. $^{\text {a }}$} & \multicolumn{2}{|c|}{ CASSCF } & \multicolumn{4}{|c|}{ B3LYP } \\
\hline & & & cc-pVDZ & aug-cc-pVDZ & cc-pVDZ & aug-cc-pVDZ & cc-pVTZ & aug-cc-pVTZ \\
\hline \multirow[t]{11}{*}{$\mathrm{A}_{1}$} & $v_{l}$ & 3090 & 3088 & 3087 & 3101 & 3100 & 3090 & 3093 \\
\hline & $v_{2}$ & 3065 & 3078 & 3077 & 3090 & 3090 & 3080 & 3083 \\
\hline & $v_{3}$ & 3018 & 3056 & 3055 & 3069 & 3070 & 3059 & 3062 \\
\hline & $v_{4}$ & 1550 & 1519 & 1508 & 1542 & 1532 & 1535 & 1534 \\
\hline & $v_{5}$ & 1481 & 1456 & 1462 & 1436 & 1426 & 1433 & 1432 \\
\hline & $v_{6}$ & 1397 & 1388 & 1388 & 1367 & 1357 & 1374 & 1373 \\
\hline & $v_{7}$ & 1167 & 1114 & 1115 & 1114 & 1118 & 1128 & 1128 \\
\hline & $v_{8}$ & 1038 & 959 & 960 & 978 & 975 & 977 & 977 \\
\hline & $v_{9}$ & 977 & 943 & 940 & 946 & 939 & 957 & 957 \\
\hline & $v_{10}$ & 813 & 766 & 762 & 782 & 780 & 780 & 780 \\
\hline & $v_{11}$ & 520 & 506 & 495 & 511 & 509 & 514 & 514 \\
\hline \multirow[t]{3}{*}{$\mathrm{A}_{2}$} & $v_{12}$ & $\ldots$ & 895 & 884 & 958 & 947 & 962 & 962 \\
\hline & $v_{13}$ & $\cdots$ & 728 & 722 & 785 & 773 & 782 & 782 \\
\hline & $v_{14}$ & $\cdots$ & 365 & 362 & 369 & 367 & 369 & 368 \\
\hline \multirow[t]{6}{*}{$\mathrm{B}_{1}$} & $v_{15}$ & 1016 & 899 & 880 & 974 & 962 & 975 & 974 \\
\hline & $v_{16}$ & 898 & 809 & 797 & 902 & 895 & 903 & 904 \\
\hline & $v_{17}$ & 784 & 721 & 703 & 780 & 776 & 780 & 782 \\
\hline & $v_{18}$ & 635 & 609 & 598 & 639 & 629 & 637 & 634 \\
\hline & $v_{19}$ & 472 & 452 & 442 & 473 & 467 & 470 & 465 \\
\hline & $v_{20}$ & $\ldots$ & 182 & 169 & 188 & 181 & 183 & 181 \\
\hline \multirow[t]{10}{*}{$\mathrm{B}_{2}$} & $v_{21}$ & 3074 & 3084 & 3083 & 3098 & 3097 & 3088 & 3090 \\
\hline & $v_{22}$ & 3054 & 3064 & 3061 & 3076 & 3076 & 3065 & 3068 \\
\hline & $v_{23}$ & 1515 & 1502 & 1497 & 1498 & 1489 & 1497 & 1496 \\
\hline & $v_{24}$ & 1441 & 1404 & 1403 & 1395 & 1387 & 1400 & 1399 \\
\hline & $v_{25}$ & 1318 & 1269 & 1274 & 1300 & 1296 & 1295 & 1296 \\
\hline & $v_{26}$ & 1266 & 1229 & 1228 & 1230 & 1233 & 1234 & 1236 \\
\hline & $v_{27}$ & 1140 & 1075 & 1075 & 1118 & 1119 & 1127 & 1126 \\
\hline & $v_{28}$ & 1072 & 1039 & 1035 & 1047 & 1049 & 1055 & 1055 \\
\hline & $v_{29}$ & 616 & 574 & 573 & 576 & 574 & 579 & 578 \\
\hline & $v_{30}$ & 446 & 440 & 444 & 429 & 427 & 431 & 432 \\
\hline
\end{tabular}

${ }^{a}$ Ref. 7. 
Table X2. Harmonic vibrational frequencies for the $\mathrm{X}^{2} \mathrm{~B}_{1}$ electronic state of $\mathrm{C}_{6} \mathrm{D}_{5} \mathrm{O}$ computed using the DFT/B3LYP and CASSCF methods. For description of the active space of CASSCF, see text. All values are given in $\mathrm{cm}^{-1}$.

\begin{tabular}{|c|c|c|c|c|c|c|c|c|}
\hline \multirow{2}{*}{ Symmetry } & \multirow{2}{*}{ Mode } & \multirow{2}{*}{ Exp. $^{a}$} & \multicolumn{2}{|c|}{ CASSCF } & \multicolumn{4}{|c|}{ B3LYP } \\
\hline & & & cc-pVDZ & aug-cc-pVDZ & cc-pVDZ & aug-cc-pVDZ & cc-pVTZ & aug-cc-pVTZ \\
\hline \multirow[t]{11}{*}{$\mathrm{A}_{1}$} & $v_{1}$ & 2331 & 2307 & 2301 & 2308 & 2308 & 2303 & 2302 \\
\hline & $v_{2}$ & 2298 & 2291 & 2286 & 2290 & 2292 & 2287 & 2287 \\
\hline & $v_{3}$ & 2284 & 2270 & 2266 & 2269 & 2272 & 2267 & 2267 \\
\hline & $v_{4}$ & 1509 & 1489 & 1483 & 1508 & 1499 & 1501 & 1498 \\
\hline & $v_{5}$ & 1467 & 1422 & 1424 & 1416 & 1404 & 1405 & 1402 \\
\hline & $v_{6}$ & 1244 & 1226 & 1223 & 1226 & 1217 & 1232 & 1230 \\
\hline & $v_{7}$ & 944 & 929 & 926 & 923 & 915 & 934 & 933 \\
\hline & $v_{8}$ & 902 & 823 & 822 & 840 & 838 & 844 & 843 \\
\hline & $v_{9}$ & 844 & 816 & 814 & 805 & 810 & 819 & 819 \\
\hline & $v_{10}$ & 724 & 705 & 704 & 708 & 710 & 712 & 712 \\
\hline & $v_{11}$ & 505 & 494 & 485 & 498 & 497 & 503 & 502 \\
\hline \multirow[t]{3}{*}{$\mathrm{A}_{2}$} & $v_{12}$ & $\ldots$ & 716 & 705 & 774 & 768 & 779 & 779 \\
\hline & $v_{13}$ & $\ldots$ & 569 & 563 & 612 & 604 & 611 & 611 \\
\hline & $v_{14}$ & $\ldots$ & 327 & 325 & 325 & 323 & 326 & 324 \\
\hline \multirow[t]{6}{*}{$\mathrm{B}_{1}$} & $v_{15}$ & 860 & 741 & 718 & 828 & 828 & 830 & 830 \\
\hline & $v_{16}$ & 775 & 708 & 690 & 773 & 767 & 775 & 775 \\
\hline & $v_{17}$ & 660 & 613 & 603 & 655 & 649 & 658 & 658 \\
\hline & $v_{18}$ & 489 & 473 & 465 & 493 & 485 & 492 & 489 \\
\hline & $v_{19}$ & 406 & 395 & 387 & 410 & 405 & 409 & 404 \\
\hline & $v_{20}$ & $\ldots$ & 172 & 161 & 177 & 170 & 173 & 171 \\
\hline \multirow[t]{10}{*}{$\mathrm{B}_{2}$} & $v_{21}$ & 2326 & 2301 & 2296 & 2301 & 2303 & 2297 & 2297 \\
\hline & $v_{22}$ & 2281 & 2277 & 2272 & 2277 & 2279 & 2274 & 2274 \\
\hline & $v_{23}$ & 1449 & 1441 & 1436 & 1439 & 1429 & 1431 & 1428 \\
\hline & $v_{24}$ & 1334 & 1292 & 1291 & 1330 & 1324 & 1321 & 1320 \\
\hline & $v_{25}$ & 1255 & 1177 & 1169 & 1247 & 1243 & 1236 & 1235 \\
\hline & $v_{26}$ & 1032 & 1025 & 1027 & 1001 & 1002 & 1021 & 1021 \\
\hline & $v_{27}$ & 836 & 818 & 816 & 819 & 821 & 830 & 828 \\
\hline & $v_{28}$ & 824 & 798 & 799 & 785 & 790 & 799 & 799 \\
\hline & $v_{29}$ & $\ldots$ & 556 & 555 & 557 & 556 & 561 & 559 \\
\hline & $v_{30}$ & 423 & 421 & 424 & 408 & 408 & 412 & 413 \\
\hline
\end{tabular}

${ }^{\mathrm{a}}$ Ref. 7. 
Table P1. Harmonic vibrational frequencies of $\mathrm{C}_{6} \mathrm{H}_{5} \mathrm{OH}$ computed using the B3LYP method.

\begin{tabular}{|c|c|c|c|c|c|c|c|c|c|c|}
\hline \multirow{2}{*}{ Symmetry } & \multirow{2}{*}{ Mode } & \multirow{2}{*}{ Exp. $^{a}$} & \multicolumn{2}{|c|}{ cc-pVDZ } & \multicolumn{2}{|c|}{ aug-cc-pVDZ } & \multicolumn{2}{|c|}{ cc-pVTZ } & \multicolumn{2}{|c|}{ aug-cc-pVTZ } \\
\hline & & & unscaled & scaled & unscaled & scaled & unscaled & scaled & unscaled & scaled \\
\hline \multirow[t]{23}{*}{$a^{\prime}$} & $v_{1}$ & 3655 & 3779 & 3654 & 3820 & 3694 & 3817 & 3687 & 3815 & 3689 \\
\hline & $v_{2}$ & 3074 & 3206 & 3100 & 3206 & 3100 & 3198 & 3089 & 3198 & 3092 \\
\hline & $v_{3}$ & 3061 & 3199 & 3094 & 3199 & 3094 & 3191 & 3083 & 3191 & 3086 \\
\hline & $v_{4}$ & 3052 & 3184 & 3079 & 3186 & 3081 & 3177 & 3069 & 3178 & 3073 \\
\hline & $v_{5}$ & 3046 & 3176 & 3071 & 3178 & 3074 & 3169 & 3061 & 3169 & 3065 \\
\hline & $v_{6}$ & 3021 & 3154 & 3050 & 3160 & 3056 & 3150 & 3043 & 3151 & 3047 \\
\hline & $v_{7}$ & 1609 & 1662 & 1607 & 1648 & 1594 & 1650 & 1594 & 1646 & 1592 \\
\hline & $v_{8}$ & 1604 & 1649 & 1594 & 1638 & 1584 & 1639 & 1583 & 1636 & 1582 \\
\hline & $v_{9}$ & 1501 & 1530 & 1479 & 1516 & 1466 & 1535 & 1483 & 1532 & 1481 \\
\hline & $v_{10}$ & 1472 & 1500 & 1450 & 1489 & 1440 & 1508 & 1456 & 1505 & 1455 \\
\hline & $v_{11}$ & 1361 & 1377 & 1332 & 1368 & 1323 & 1377 & 1330 & 1375 & 1329 \\
\hline & $v_{12}$ & 1344 & 1350 & 1306 & 1347 & 1303 & 1350 & 1304 & 1348 & 1303 \\
\hline & $v_{13}$ & 1261 & 1300 & 1257 & 1272 & 1230 & 1287 & 1243 & 1280 & 1237 \\
\hline & $v_{14}$ & 1197 & 1199 & 1160 & 1190 & 1151 & 1195 & 1155 & 1192 & 1153 \\
\hline & $v_{15}$ & 1167 & 1178 & 1139 & 1181 & 1142 & 1194 & 1153 & 1191 & 1152 \\
\hline & $v_{16}$ & 1150 & 1165 & 1127 & 1167 & 1129 & 1179 & 1139 & 1176 & 1137 \\
\hline & $v_{17}$ & 1070 & 1090 & 1054 & 1089 & 1053 & 1097 & 1059 & 1095 & 1059 \\
\hline & $v_{18}$ & 1026 & 1044 & 1009 & 1041 & 1007 & 1047 & 1011 & 1046 & 1011 \\
\hline & $v_{19}$ & 999 & 1009 & 975 & 1005 & 972 & 1019 & 984 & 1019 & 985 \\
\hline & $v_{20}$ & 810 & 831 & 804 & 824 & 797 & 832 & 803 & 830 & 803 \\
\hline & $v_{21}$ & 618 & 629 & 608 & 627 & 607 & 635 & 613 & 633 & 612 \\
\hline & $v_{22}$ & 526 & 533 & 516 & 532 & 514 & 538 & 520 & 537 & 519 \\
\hline & $v_{23}$ & 410 & 403 & 390 & 402 & 389 & 407 & 393 & 406 & 392 \\
\hline \multirow[t]{10}{*}{$a^{\prime \prime}$} & $v_{24}$ & 973 & 995 & 962 & 991 & 958 & 993 & 960 & 1002 & 969 \\
\hline & $v_{25}$ & 956 & 970 & 938 & 968 & 936 & 972 & 939 & 980 & 948 \\
\hline & $v_{26}$ & 881 & 895 & 865 & 897 & 867 & 900 & 869 & 906 & 876 \\
\hline & $v_{27}$ & 823 & 826 & 798 & 822 & 795 & 828 & 800 & 832 & 805 \\
\hline & $v_{28}$ & 752 & 768 & 743 & 765 & 739 & 769 & 743 & 773 & 748 \\
\hline & $v_{29}$ & 687 & 704 & 681 & 704 & 681 & 707 & 683 & 704 & 681 \\
\hline & $v_{30}$ & 503 & 521 & 504 & 515 & 498 & 518 & 501 & 516 & 499 \\
\hline & $v_{31}$ & 420 & 423 & 409 & 421 & 407 & 424 & 409 & 423 & 409 \\
\hline & $v_{32}$ & 310 & 368 & 355 & 359 & 347 & 356 & 344 & 354 & 342 \\
\hline & $v_{33}$ & 242 & 233 & 225 & 229 & 222 & 231 & 223 & 230 & 222 \\
\hline
\end{tabular}

${ }^{a}$ Ref. 52. 
Table P2. Harmonic vibrational frequencies of $\mathrm{C}_{6} \mathrm{D}_{5} \mathrm{OD}$ computed using the B3LYP method.

\begin{tabular}{|c|c|c|c|c|c|c|c|c|c|c|}
\hline \multirow{2}{*}{ Symmetry } & \multirow{2}{*}{ Mode } & \multirow{2}{*}{ Exp. ${ }^{a}$} & \multicolumn{2}{|c|}{ cc-pVDZ } & \multicolumn{2}{|c|}{ aug-cc-pVDZ } & \multicolumn{2}{|c|}{ cc-pVTZ } & \multicolumn{2}{|c|}{ aug-cc-pVTZ } \\
\hline & & & unscaled & scaled & unscaled & scaled & unscaled & scaled & unscaled & scaled \\
\hline \multirow[t]{23}{*}{$a^{\prime}$} & $v_{1}$ & 2655 & 2751 & 2668 & 2781 & 2700 & 2778 & 2698 & 2777 & 2697 \\
\hline & $v_{2}$ & 2294 & 2378 & 2307 & 2378 & 2309 & 2370 & 2302 & 2370 & 2301 \\
\hline & $v_{3}$ & 2286 & 2369 & 2297 & 2368 & 2300 & 2361 & 2293 & 2361 & 2292 \\
\hline & $v_{4}$ & 2277 & 2354 & 2284 & 2356 & 2287 & 2348 & 2280 & 2349 & 2281 \\
\hline & $v_{5}$ & 2256 & 2342 & 2272 & 2344 & 2276 & 2338 & 2270 & 2338 & 2270 \\
\hline & $v_{6}$ & 2246 & 2328 & 2258 & 2333 & 2265 & 2325 & 2257 & 2326 & 2258 \\
\hline & $v_{7}$ & 1585 & 1631 & 1582 & 1615 & 1568 & 1614 & 1568 & 1610 & 1563 \\
\hline & $v_{8}$ & 1567 & 1613 & 1564 & 1603 & 1557 & 1601 & 1555 & 1598 & 1552 \\
\hline & $v_{9}$ & 1391 & 1437 & 1394 & 1416 & 1375 & 1426 & 1384 & 1421 & 1380 \\
\hline & $v_{10}$ & 1351 & 1385 & 1343 & 1373 & 1333 & 1382 & 1342 & 1380 & 1340 \\
\hline & $v_{11}$ & 1292 & 1355 & 1314 & 1347 & 1308 & 1330 & 1291 & 1328 & 1289 \\
\hline & $v_{12}$ & 1178 & 1221 & 1185 & 1199 & 1164 & 1213 & 1178 & 1207 & 1172 \\
\hline & $v_{13}$ & 1056 & 1063 & 1032 & 1060 & 1029 & 1080 & 1049 & 1079 & 1048 \\
\hline & $v_{14}$ & 957 & 970 & 941 & 965 & 937 & 977 & 949 & 977 & 948 \\
\hline & $v_{15}$ & 905 & 916 & 889 & 908 & 882 & 914 & 888 & 911 & 884 \\
\hline & $v_{16}$ & 874 & 880 & 854 & 877 & 852 & 888 & 862 & 886 & 860 \\
\hline & $v_{17}$ & 855 & 846 & 820 & 846 & 822 & 857 & 832 & 856 & 831 \\
\hline & $v_{18}$ & 834 & 835 & 810 & 838 & 814 & 848 & 823 & 847 & 822 \\
\hline & $v_{19}$ & 811 & 817 & 793 & 820 & 796 & 830 & 806 & 829 & 805 \\
\hline & $v_{20}$ & 750 & 759 & 737 & 756 & 734 & 764 & 742 & 762 & 740 \\
\hline & $v_{21}$ & 593 & 602 & 584 & 601 & 584 & 608 & 591 & 606 & 589 \\
\hline & $v_{22}$ & 509 & 514 & 499 & 513 & 498 & 519 & 504 & 518 & 503 \\
\hline & $v_{23}$ & 367 & 367 & 356 & 366 & 356 & 371 & 360 & 369 & 359 \\
\hline \multirow[t]{10}{*}{$a^{\prime \prime}$} & $v_{24}$ & 801 & 824 & 799 & 839 & 815 & 825 & 801 & 839 & 815 \\
\hline & $v_{25}$ & 786 & 786 & 763 & 788 & 765 & 789 & 766 & 797 & 773 \\
\hline & $v_{26}$ & 753 & 769 & 746 & 770 & 748 & 773 & 750 & 777 & 754 \\
\hline & $v_{27}$ & 655 & 646 & 627 & 641 & 623 & 647 & 629 & 650 & 631 \\
\hline & $v_{28}$ & 623 & 636 & 617 & 629 & 610 & 636 & 618 & 638 & 619 \\
\hline & $v_{29}$ & 551 & 562 & 545 & 557 & 541 & 564 & 547 & 559 & 543 \\
\hline & $v_{30}$ & 432 & 443 & 430 & 436 & 424 & 441 & 428 & 440 & 427 \\
\hline & $v_{31}$ & 358 & 369 & 358 & 366 & 355 & 369 & 359 & 368 & 357 \\
\hline & $v_{32}$ & 235 & 274 & 266 & 268 & 260 & 267 & 259 & 265 & 258 \\
\hline & $v_{33}$ & 203 & 213 & 206 & 210 & 204 & 210 & 204 & 209 & 203 \\
\hline
\end{tabular}

${ }^{\mathrm{a}}$ Ref. 53 
Table P3. Harmonic vibrational frequencies of $\mathrm{C}_{6} \mathrm{H}_{5} \mathrm{OH}$ computed using the CASSCF method.

\begin{tabular}{|c|c|c|c|c|c|c|}
\hline \multirow{2}{*}{ Symmetry } & \multirow{2}{*}{ Mode } & \multirow{2}{*}{ Exp. $^{\text {a }}$} & \multicolumn{2}{|c|}{ cc-pVDZ } & \multicolumn{2}{|c|}{ aug-cc-pVDZ } \\
\hline & & & unscaled & scaled & unscaled & scaled \\
\hline \multirow[t]{23}{*}{$a^{\prime}$} & $v_{l}$ & 3655 & 4161 & 3807 & 4185 & 3838 \\
\hline & $v_{2}$ & 3074 & 3370 & 3083 & 3371 & 3091 \\
\hline & $v_{3}$ & 3061 & 3361 & 3075 & 3351 & 3073 \\
\hline & $v_{4}$ & 3052 & 3347 & 3063 & 3339 & 3062 \\
\hline & $v_{5}$ & 3046 & 3336 & 3052 & 3327 & 3051 \\
\hline & $v_{6}$ & 3021 & 3317 & 3035 & 3310 & 3035 \\
\hline & $v_{7}$ & 1609 & 1757 & 1608 & 1777 & 1630 \\
\hline & $v_{8}$ & 1604 & 1739 & 1591 & 1732 & 1588 \\
\hline & $v_{9}$ & 1501 & 1633 & 1495 & 1657 & 1519 \\
\hline & $v_{10}$ & 1472 & 1596 & 1460 & 1591 & 1459 \\
\hline & $v_{11}$ & 1361 & 1462 & 1338 & 1459 & 1338 \\
\hline & $v_{12}$ & 1344 & 1381 & 1264 & 1350 & 1238 \\
\hline & $v_{13}$ & 1261 & 1356 & 1241 & 1278 & 1172 \\
\hline & $v_{14}$ & 1197 & 1270 & 1162 & 1254 & 1150 \\
\hline & $v_{15}$ & 1167 & 1251 & 1145 & 1231 & 1129 \\
\hline & $v_{16}$ & 1150 & 1180 & 1080 & 1183 & 1085 \\
\hline & $v_{17}$ & 1070 & 1144 & 1047 & 1141 & 1046 \\
\hline & $v_{18}$ & 1026 & 1092 & 1000 & 1074 & 985 \\
\hline & $v_{19}$ & 999 & 1067 & 976 & 1016 & 932 \\
\hline & $v_{20}$ & 810 & 871 & 797 & 902 & 827 \\
\hline & $v_{21}$ & 618 & 664 & 607 & 663 & 608 \\
\hline & $v_{22}$ & 526 & 563 & 515 & 556 & 510 \\
\hline & $v_{23}$ & 410 & 430 & 393 & 428 & 392 \\
\hline \multirow[t]{10}{*}{$a^{\prime \prime}$} & $v_{24}$ & 973 & 997 & 912 & 976 & 895 \\
\hline & $v_{25}$ & 956 & 973 & 890 & 958 & 878 \\
\hline & $v_{26}$ & 881 & 893 & 817 & 884 & 811 \\
\hline & $v_{27}$ & 823 & 831 & 760 & 826 & 757 \\
\hline & $v_{28}$ & 752 & 767 & 702 & 752 & 690 \\
\hline & $v_{29}$ & 687 & 714 & 654 & 686 & 629 \\
\hline & $v_{30}$ & 503 & 538 & 492 & 516 & 473 \\
\hline & $v_{31}$ & 420 & 435 & 398 & 432 & 396 \\
\hline & $v_{32}$ & 310 & 288 & 263 & 272 & 249 \\
\hline & $v_{33}$ & 242 & 247 & 226 & 240 & 220 \\
\hline
\end{tabular}

${ }^{\mathrm{a}}$ Ref. 52. 
Table P4. Harmonic vibrational frequencies of $\mathrm{C}_{6} \mathrm{D}_{5} \mathrm{OD}$ computed using the CASSCF method.

\begin{tabular}{|c|c|c|c|c|c|c|}
\hline \multirow{2}{*}{ Symmetry } & \multirow{2}{*}{ Mode } & \multirow{2}{*}{ Exp. $^{a}$} & \multicolumn{2}{|c|}{ cc-pVDZ } & \multicolumn{2}{|c|}{ aug-cc-pVDZ } \\
\hline & & & unscaled & scaled & unscaled & scaled \\
\hline \multirow[t]{23}{*}{$a^{\prime}$} & $v_{l}$ & 2655 & 3040 & 2794 & 3057 & 2819 \\
\hline & $v_{2}$ & 2294 & 2506 & 2303 & 2504 & 2308 \\
\hline & $v_{3}$ & 2286 & 2497 & 2295 & 2489 & 2295 \\
\hline & $v_{4}$ & 2277 & 2483 & 2282 & 2480 & 2286 \\
\hline & $v_{5}$ & 2256 & 2470 & 2270 & 2465 & 2273 \\
\hline & $v_{6}$ & 2246 & 2457 & 2258 & 2454 & 2262 \\
\hline & $v_{7}$ & 1585 & 1717 & 1578 & 1713 & 1580 \\
\hline & $v_{8}$ & 1567 & 1692 & 1555 & 1685 & 1553 \\
\hline & $v_{9}$ & 1391 & 1524 & 1400 & 1521 & 1403 \\
\hline & $v_{10}$ & 1351 & 1452 & 1334 & 1445 & 1332 \\
\hline & $v_{11}$ & 1292 & 1299 & 1194 & 1255 & 1158 \\
\hline & $v_{12}$ & 1178 & 1256 & 1154 & 1247 & 1149 \\
\hline & $v_{13}$ & 1056 & 1149 & 1056 & 1146 & 1057 \\
\hline & $v_{14}$ & 957 & 1026 & 943 & 1015 & 935 \\
\hline & $v_{15}$ & 905 & 986 & 906 & 980 & 903 \\
\hline & $v_{16}$ & 874 & 932 & 857 & 928 & 855 \\
\hline & $v_{17}$ & 855 & 903 & 830 & 904 & 833 \\
\hline & $v_{18}$ & 834 & 893 & 820 & 894 & 824 \\
\hline & $v_{19}$ & 811 & 873 & 802 & 839 & 774 \\
\hline & $v_{20}$ & 750 & 801 & 736 & 817 & 754 \\
\hline & $v_{21}$ & 593 & 637 & 585 & 628 & 579 \\
\hline & $v_{22}$ & 509 & 543 & 499 & 540 & 497 \\
\hline & $v_{23}$ & 367 & 393 & 361 & 389 & 358 \\
\hline \multirow[t]{10}{*}{$a^{\prime \prime}$} & $v_{24}$ & 801 & 808 & 743 & 782 & 721 \\
\hline & $v_{25}$ & 786 & 783 & 720 & 767 & 707 \\
\hline & $v_{26}$ & 753 & 762 & 701 & 743 & 685 \\
\hline & $v_{27}$ & 655 & 649 & 597 & 645 & 595 \\
\hline & $v_{28}$ & 623 & 646 & 593 & 632 & 583 \\
\hline & $v_{29}$ & 551 & 576 & 529 & 568 & 524 \\
\hline & $v_{30}$ & 432 & 463 & 425 & 445 & 410 \\
\hline & $v_{31}$ & 358 & 384 & 353 & 381 & 351 \\
\hline & $v_{32}$ & 235 & 240 & 221 & 233 & 215 \\
\hline & $v_{33}$ & 203 & 203 & 187 & 193 & 178 \\
\hline
\end{tabular}

${ }^{\mathrm{a}}$ Ref. 53. 
Table K. Total energies, vertical excitation energies, and adiabatic excitation energies for the $\mathrm{X}^{2} \mathrm{~B}_{1}$ and $\mathrm{A}^{2} \mathrm{~B}_{2}$ electronic states of phenoxyl radical computed using the CASSCF methods. All CASSCF calculations use the same $\left(0 a_{1}, 2 a_{2}, 5 b_{1}, 1 b_{2}\right)$ active space described in details in text. $\Delta E_{H}^{0-0}$ denotes the $0-0$ excitation energy for $\mathrm{C}_{6} \mathrm{H}_{5} \mathrm{O}$ and $\Delta E_{D}^{0-0}$, for $\mathrm{C}_{6} \mathrm{D}_{5} \mathrm{O}$. Total energies are given in hartree and excitation energies, in $\mathrm{eV}$.

\begin{tabular}{|c|c|c|c|c|c|c|c|}
\hline \multirow{2}{*}{ Basis set } & \multicolumn{3}{|c|}{ Total energies } & \multicolumn{4}{|c|}{$\mathrm{A}^{2} \mathrm{~B}_{2} \leftarrow \mathrm{X}^{2} \mathrm{~B}_{1}$ excitation energies } \\
\hline & $\mathrm{X}^{2} \mathrm{~B}_{1}$ & $\mathrm{~A}^{2} \mathrm{~B}_{2}$ (vertical) & $\mathrm{A}^{2} \mathrm{~B}_{2}$ (adiabatic) & vertical & adiabatic & $\Delta E_{H}^{0-0}$ & $\Delta E_{D}^{0-0}$ \\
\hline \multicolumn{8}{|c|}{ CASSCF } \\
\hline cc-pVDZ & -305.059491 & -305.011327 & -305.029196 & 1.311 & 0.824 & 0.823 & 0.821 \\
\hline aug-cc-pVDZ & -305.071003 & -305.021342 & -305.040081 & 1.351 & 0.842 & 0.838 & 0.837 \\
\hline cc-pVTZ & -305.137004 & -305.087473 & -305.106565 & 1.348 & 0.828 & 0.825 & 0.823 \\
\hline aug-cc-pVTZ & -305.139698 & -305.089904 & -305.109088 & 1.355 & 0.833 & 0.830 & 0.828 \\
\hline
\end{tabular}

